\title{
Efectividad de la Naltrexona entre pacientes alcohólicos
}

\author{
Torres Hernández M.A.*; Arias Horcajadas F**; Ochoa Mangado E.*** \\ *Unidad de Alcoholismo y Toxicomanías. Salud Mental Área 8. 46900 TORRENT (Valencia) \\ ${ }^{*}$ Centro de Salud Mental de Santa Elena. Zamora. \\ *** Servicio de Psiquiatría. Hospital Ramón y Cajal. Madrid \\ Enviar correspondencia: \\ Miguel Ángel Torres Hernández. Unidad de Alcoholismo y Toxicomanías. Salud Mental Área 8. Hernández Malillos, 14, bajo. \\ 46900 TORRENT (Valencia) MTORRE@mail.ono.es
}

\section{Resumen:}

Tras un período de introducción de la naltrexona como indicación para la reducción del craving y mantenimiento de la abstinencia entre alcohólicos. Se han querido confirmar estos aspectos a través de un estudio entre pacientes alcohólicos de diferentes centros de tratamiento españoles.

Objetivo: valorar la efectividad de naltrexona entre alcohólicos para ver si se obtiene un mayor mantenimiento de la abstinencia y valorar si no se pierde el control aún habiendo consumido alcohol.

Material y métodos: estudio abierto, no controlado, prospectivo y multicéntrico en 42 centros de tratamiento del alcoholismo de España, con seguimiento de 6 meses por paciente. Se seleccionó una muestra de 234 pacientes, de los que finalizaron 173 con todo el seguimiento. Se establecieron criterios de inclusión y exclusión, así como criterios de recaída y cumplimiento de tratamiento y las técnicas de los mismos, con cuestionario elaborado ad hoc junto con escalas como la EEAG, EuropASI, EIDA, CIWA-Ar, HDS, etc.

Resultados: $86,1 \%$ de varones, edad $43,6 \pm 10,9$ años. duración del consumo en años 19,5. EEAG: 63,5 de mediana. Bebedores diarios $77,5 \%$. Patrón de consumo solitario y social 48,6\%. Dosis media 150 gr./día. Area Alcohol EuropASI 7. EIDA

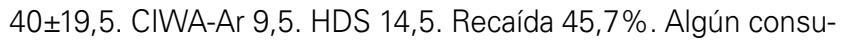
mo 59,4\%. No recaídas no incluyendo abandonos 61,9\%. Mejoría moderada o marcada según paciente $86 \%$ y según médico $82,3 \%$. No deseo de consumo $58 \%$ en los abstinentes y entre los que bebieron $39 \%$.

Como conclusión se puede confirmar una baja tasa de recaídas y mejoría en el mantenimiento de la abstinencia.

Palabras clave: alcoholismo, naltrexona, recaídas, efectividad de tratamiento.

\section{Abstract:}

A spanish open-label, multicentre, post-marketing surveillance study was made in alcoholic outpatients.

Objectives: To evaluate the effectivity of Naltrexone in alcoholics in order to obtain retention rate and alcohol consumptionfree-time.

Material and methods: A sample of 234 alcoholic outpatients was obtain from 42 Spanish centres. Follow-up was completed by 173. Inclusion and exclusion criteria, relapse criteria and treatment compliance and their techniques were stablished.

Evaluation was made using and "ad hoc" questionnaire together with EEAG, EuropASI, EIDA, CIWA-Ar, HDS, etc.

Results: $86.1 \%$ were males, mean age $43.6 \pm 10.9$ years. Duration of consumption 19.5 years. EEAG: median 63.5. Daily drinkers $(77,5 \%)$. Pattern of solitary and social drinkers (48.6\%), mean dose $150 \mathrm{gr} /$ day. Area of alcohol on EuropASI: 7. EIDA: 40 \pm 19.5. CIWA-Ar: 9.5. HDS: 14.5. Relapse (45.5\%). Some consumption: $59.4 \%$ in those abstinent. Non relapses, not including those who had abandoned treatment (61.9\%). Moderate or marked improvement according to patient (86\%), according to clinician $82.3 \%$. No desire to consume: $58 \%$ in abstainers and $39 \%$ in drinkers.

We found a low rate of relapses, and improvement in the maintenance of abstinence.

Key words alcoholism, naltrexone, relapse, relapse, treatment effectivity.

\section{INTRODUCCIÓN}

A lo largo de la historia más reciente se están buscando más medidas terapéuticas para el alcoholismo debido a su escasez, a pesar de los años que lleva conviviendo dicha enfermedad con nosotros y nuestra cultura. La psicoterapia aislada, puede derivar hacia una recaída con más facilidad que si la acompañamos de otras medidas (Nathan, 1986)'. Venimos utilizando tres tipos de agentes farmacológi- 
cos: Ios que producen efectos aversivos, los que bloquean las propiedades reforzantes positivas o negativas y que llevan a una menor motivación para beber y, por último, los que disminuyen el deseo de beber. Estos dos últimos aspectos pueden ser próximos pero no necesariamente idénticos (Froehlich y Li, 1993) ${ }^{2}$. Los aversivos, frente a placebo en estudios a doble ciego, no han demostrado siempre su eficacia absoluta (Fuller y Roth, 1979³ , Fuller et al. 1986²).

Altshuler et al. en $1980^{5}$, comprobaron en monos que la naltrexona disminuía la autoadministración intravenosa de alcohol en éstos, reduciendo su acción reforzante, disminuyendo su potencial de abuso, siendo el efecto dosis-dependiente. Asimismo, desde los estudios de Gianoulakis en $1993^{6}$, se empezó a elaborar la teoría del papel del sistema opioide endógeno en la vulnerabilidad al desarrollo del alcoholismo. También Aguirre et al. en $1990^{7}$ y Gianoulakis et al. en $1996^{8}$ determinaron que las personas con dependencia alcohólica tenían más bajo nivel de beta endorfina plasmática, igual que los sujetos no alcohólicos de alto riesgo. El alcohol incrementa de una forma dosisdependiente los niveles de beta endorfina en el grupo de alto riesgo, pero no en los de bajo riesgo. La mayor activación opioide, la de mayor refuerzo y, la que produce más placer por la activación del alcohol, seguramente por factores genéticos, incrementa la probabilidad del desarrollo de alcoholismo (Froehlich 1995) $)^{9}$.

Otros autores como Swift $1995^{10}$ indican que la naltrexona disminuye los efectos positivos y aumenta los sedativos y negativos, intensifican ciertos signos de intoxicación alcohólica y, tras una pequeña cantidad de alcohol experimentaban los efectos de una ingesta elevada y preferían la ingesta de placebo, reconociendo que es difícil diferenciar si el efecto primario es un descenso de los efectos positivos o un incremento de los aversivos.

Las propuestas derivadas de ello, según el criterio de autores como O'Mara y Wesley $1994^{11}$, Volpicelli et al. $1992^{12}$ indican que un agente ideal para el tratamiento del alcoholismo debe cumplir los siguientes criterios: 1. Disminuir la apetencia y el deseo por el alcohol. 2. Reducir la motivación a beber por disminuir las sensaciones placenteras asociadas al consumo. 3 . Carecer de efectos adversos físicos relevantes o potencial adictivo. 4. Evitar interacciones con el alcohol. De los fármacos últimamente empleados, la naltrexona parece adecuarse a estas exigencias.

Los estudios clásicos de Volpicelli ${ }^{13}$ y $\mathrm{O}^{\prime} \mathrm{Malley}^{14}$ en 1992, reforzaron esta opinión al obtener resultados similares, recomendando el uso de naltrexona como tratamiento complementario a otras modalidades psicoterapéuticas, resultando efectivo en aquellos sujetos que mantenían algún consumo de alcohol, disminuyendo el deseo de sucesivas ingestas. Volpicelli13 incluyó 70 alcohólicos, tratados con naltrexona
(50 mg diarios) o placebo durante 12 semanas con diseño doble ciego. Existieron diferencias significativas en el porcentaje de recaídas durante el tratamiento $(23 \%$ frente a $54,3 \%)$ a favor del grupo de naltrexona. Destaca que el $50 \%$ de los sujetos que tomaron naltrexona recayeron tras probar el alcohol, inferior al 95\% de recaídas presentes en el grupo placebo tras dicho consumo.

O’Malley14 revisó los datos de 97 pacientes alcohólicos, en estudio doble ciego, comparando también dos técnicas psicoterapéuticas. El porcentaje de sujetos que bebieron durante el tratamiento fue inferior con el uso de naltrexona, al igual que el consumo intenso (25\% frente al $60 \%$ ). Al igual que el grupo de Volpicelli, observaron que entre aquellos sujetos que consumieron algo de alcohol, los que tomaban naltrexona tenían menor riesgo de recaer.

Volpicelli15, de nuevo en 1997, en 97 dependientes del alcohol, en condiciones más similares a la clínica habitual, con menos estructuración del programa psicoterapéutico, usando el consejo individual sin otras medidas psicoterapéuticas, obtuvo resultados inferiores. Las recaídas fueron del $53 \%$ en el grupo placebo y el 35 en el de naltrexona en el análisis de los sujetos con intención de tratar $(p=0,07)$.

Ya en España, Martínez et al $^{16}$ en 1995, en un estudio abierto con 20 alcohólicos tratados con naltrexona (50 mg/día) durante 12 semanas y escaso apoyo psicoterapéutico, señalan que 13 de los 20 sujetos se consideraron respondedores ya que normalizaron los niveles de transferrina deficiente en carbohidratos (CDT, Transferrina Deficiente en Carbohidratos menor de $20 \mathrm{U} / \mathrm{I}$ ). Landabaso et al. ${ }^{17}$ en 1997, en un grupo de 30 alcohólicos con mala respuesta previa al tratamiento con aversivos, observan que la adición de $25 \mathrm{mg}$ diarios de naltrexona mejora la eficacia durante el seguimiento de 6 meses. Por lo tanto, los pocos trabajos con naltrexona para el tratamiento del alcoholismo, es lo que hizo que nos planteáramos un estudio sobre la eficacia de la naltrexona en el tratamiento de los pacientes alcohólicos y valorar su eficacia.

\section{Objetivos}

Valorar la efectividad de la naltrexona: para el mantenimiento de la abstinencia en alcohólicos, para conseguir no perder el control aún habiéndose consumido alcohol.

\section{PACIENTES, MATERIAL Y MÉTODOS}

Estudio abierto, no controlado, prospectivo y multicéntrico realizado en una muestra de pacientes de 42 centros de tratamiento para el alcoholismo en España. Inclusión de pacientes: se inició en octubre-noviembre 
Tabla 1. Aspectos sociodemográficos y clínicos

Edad

$43,6 \pm 10,96$. Rango (22-71)

\begin{tabular}{|c|c|}
\hline $\begin{array}{l}\text { Sexo } \\
\text { Varones } \\
\text { Mujeres }\end{array}$ & $\begin{array}{l}149(86,1 \%) \\
85(13,9 \%)\end{array}$ \\
\hline Ausencia de patología médica & $108(62,4 \%)$ \\
\hline E.E.A.G. & Mediana: 63,5 \\
\hline Duración del consumo en años & Mediana: 19,5 \\
\hline Frecuencia de consumo: diario & $134(77,5 \%)$ \\
\hline $\begin{array}{l}\text { Patrón de consumo: } \\
\text { Solitario } \\
\text { Social } \\
\text { Mixto }\end{array}$ & $\begin{array}{l}44(25,4 \%) \\
45(26 \%) \\
84(48,6 \%)\end{array}$ \\
\hline Dosis promedio en UBE & Mediana: $15 . \approx 150$ gramos. \\
\hline Sujetos con períodos de abstinencia previos (> 1 mes) & $103(61,3 \%)$ \\
\hline Craving (0-9) & Mediana: 7 \\
\hline $\begin{array}{l}\text { EUROPASI } \\
\text { Área Médica } \\
\text { Laboral } \\
\text { Alcohol } \\
\text { Drogas } \\
\text { Legal } \\
\text { Familiar } \\
\text { Psiquiátrica }\end{array}$ & $\begin{array}{l}\text { Mediana:3 } \\
\text { Mediana: } 5 \\
\text { Mediana: } 7 \\
\text { Mediana: } 0 \\
\text { Mediana: } 0 \\
\text { Mediana: } 6 \\
\text { Mediana: } 2\end{array}$ \\
\hline EIDA total y rango & 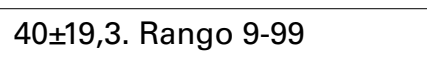 \\
\hline C.I.W.A.-Ar & Mediana: 9,5 \\
\hline Hamilton Depression Scale & 14,5 \pm 8. Rango: 0-40. \\
\hline
\end{tabular}

\begin{tabular}{|c|c|c|c|c|}
\hline \multirow[b]{2}{*}{ RECAÍDAS O ABSTINENCIA } & \multicolumn{2}{|c|}{ Tabla 2.} & \multirow[b]{2}{*}{$\begin{array}{l}\text { DATOS } \\
n=173\end{array}$} & \multirow[b]{2}{*}{$\begin{array}{c}\text { FINALES } \\
P<0,05\end{array}$} \\
\hline & $\begin{array}{c}\text { CON DATOS } \\
\mathrm{N}=234\end{array}$ & $\begin{array}{l}\text { INCOMPLETOS } \\
(P<0,05)\end{array}$ & & \\
\hline RECAÍDA SÍ & 140 & $59,8 \%$ & 79 & $45,7 \%$ \\
\hline NO RECAÍDA & 94 & $40,1 \%$ & 94 & $54,3 \%$ \\
\hline
\end{tabular}

de 1998, con un período máximo de inclusión de unos 6 meses.

El seguimiento de los pacientes se hará, al menos de seis meses para cada paciente. La recogida de datos finalizó en el segundo trimestre de 1999. El análisis de los mismos se inició en el tercer trimestre de 1999.

\section{Sujetos del estudio}

Se seleccionó una muestra de 294 pacientes, repartidos entre unos 40 investigadores que aportaron cada uno 7 pacientes al estudio.

Selección de sujetos.

Criterios de inclusión: se incluyeron pacientes mayores de 18 años con criterios de Dependencia al alcohol según criterios CIE-10 que no estuvieran en tratamiento de deshabituación en el momento de seleccionarlos, que solicitaran iniciar dicho tratamiento, dando su consentimiento a participar en el estudio y no fuera esperable la pérdida de su seguimiento durante los seis meses por causas ajenas a su dependencia (por ej. cambio de domicilio).

Criterios de exclusión: Embarazo, mujeres en edad fértil sin métodos anticonceptivos adecuados y lactantes; sujetos en tratamiento para la deshabituación alcohólica en la actualidad o con más de un mes de abstinencia por alcohol; sujetos en tratamiento psicofarmacológico por otros trastornos y cuyo tratamiento no pueda suprimirse (antidepresivos, neurolépticos, litio, carbamacepina); enfermedades orgánicas graves (hepatopatías o nefropatías severas); Dependencia o 
Tabla 3

RECAÍDAS O NO SIN INCLUIR ABANDONOS MUESTRA GLOBAL $(n=200)$ PACIENTES FINALIZADOS ( $n=165)$ NO SIGNIFICATIVO

$\begin{array}{llc}\text { RECAÍDA NO INCLUYENDO ABANDONOS } & \text { 77-(38,5\%) } & \text { 63-(38,1\%) } \\ \text { NO RECAÍDA NO INCLUYENDO ABANDONOS } & 123-(61,5 \%) & \mathbf{1 0 2}-(\mathbf{6 1 , 9 \% )}\end{array}$

\begin{tabular}{|lcc|}
\hline \multicolumn{3}{c|}{ Tabla $\mathbf{4}$} \\
IMPRESIÓN CLÍNICA GLOBAL & SEGÚN PACIENTE $(\mathrm{n}=144)$ & \\
\hline MEJORÍA MARCADA & $95-(65,9 \%)$ & SEGÚN CLÍNICO $(\mathrm{n}=153)$ \\
MEJORÍA MODERADA & $29-(20,1 \%)$ & $90-(58,8 \%)$ \\
MEJORÍA MÍNIMA & $7-(4,8 \%)$ & $36-(23,5 \%)$ \\
SIN CAMBIO & $11-(7,6 \%)$ & $15-(9,8 \%)$ \\
EMPEORAMIENTO LEVE & 0 & $8-(5,2 \%)$ \\
EMPEORAMIENTO MODERADO & $1-(0,6 \%)$ & $1-(0,6 \%)$ \\
EMPEORAMIENTO MARCADO & $1-(0,6 \%)$ & $2-(1,3 \%)$ \\
& & $1-(0,6 \%)$ \\
\hline Coeficiente de correlación Spearman $\mathbf{r = 0 , 7 2 7 9 . ~ P < 0 , 0 0 1 . ~ S . e . : ~} \mathbf{0 , 0 5}$. & \\
\hline
\end{tabular}

abuso de opiáceos o cocaína, así como de benzodiacepinas.

\section{Descripción del tratamiento}

1) Tratamiento de desintoxicación: como tratamiento de desintoxicación de alcohol se ha usado el habitual en la unidad que trabaje el investigador (ambulatorio u hospitalario). 2) Inicio del tratamiento: introducción del tratamiento con naltrexona y en el estudio cuando el sujeto tome el primer comprimido de naltrexona, tras una abstinencia superior a 5 días. En ese momento se realiza la entrevista basal. 3) Medicación del estudio: se utilizó naltrexona, diariamente, en una sola toma. Administrada bajo supervisión de la pareja o la familia. Se administró naltrexona, al menos 6 meses. 4) Dosis de naltrexona: se emplearon $50 \mathrm{mg}$ de naltrexona diarios. Solamente en el caso de aparición de efectos adversos molestos con dicha dosis se disminuyó a 25 mg por día. 5) Medicaciones complementarias. Se permitió durante el seguimiento los siguientes psicofármacos: hipnóticos, vitaminas y cualquier otra medicación usada para alguna patología orgánica de base sin efectos psicoactivos. No se han usado: benzodiacepinas no hipnóticas, antidepresivos, aversivos de alcohol, acamprosato, litio o carbamacepina. En los casos en que se tuvo que emplear alguno de dichos fármacos, el paciente fue excluido del estudio. 6) Asociación con técnicas psicoterapéuticas: la efectividad de la naltrexona en alcoholismo sin técnicas psicoterapéuticas complementarias es desconocida. Se parte de la hipótesis de que con un apoyo psicoterapéutico la naltrexona es más eficaz. Es decir, la naltrexona debe formar parte de un abordaje integral del alcoholismo. 7) Cumplimiento del tratamiento: se consideró imprescindible el cumplimiento del tratamiento con naltrexona para valorar su eficacia. Durante el seguimiento se valoró el cumplimiento del tratamiento con naltrexona mediante preguntas dirigidas al paciente y al acompañante. Asimismo, se anotaron en el Cuaderno de Recogida de Datos las faltas a las visitas programadas. 8) Criterios para valorar la recaída: Cuando en alguna de las visitas ha habido un consumo $\geq 5$ días. Cuando las Unidades Básicas de Bebida Estándar (UBE) determinada por Socidrogalcohol UBE/día $\geq 5$ (50 gr. Aproximadamente). Cantidades consumida por ocasión $\geq 5$ UBEs (50 gramos). Abandono por causas desconocidas.

Desarrollo del estudio: Se valoraron cuatro visitas por paciente durante seis meses de seguimiento. La visita basal es la que se inicia el tratamiento con naltrexona, una vez cumplido el tratamiento de desintoxicación y pasados al menos 5 días de abstinencia. Se valoraron los criterios de dependencia de la CIE-10, el cuestionario EIDA (Escala de Intensidad de la Dependencia Alcohólica), variables sociodemográficas y de consumo y una entrevista con el EuropASI (versión europea del Addiction Severity Index) y análisis de sangre.

Las visitas segunda y tercera se realizaron al primer y tercer mes, respectivamente, de tratamiento, valoran el consumo de alcohol, cumplimiento y tolerancia con el tratamiento.

La última visita se llevó a cabo al sexto mes, donde se volvió a aplicar el EuropASI y una valoración global de la eficacia de tratamiento. 
Tabla 5 Cambios manifestados por los pacientes en relación con el alcohol

Deseo intenso de consumo entre los que

bebieron y no bebieron $(n=169)(p=0,04)$

NO DESEO DE CONSUMO

SÍ DESEO DE CONSUMO
NO BEBIERON

$8(58 \%)$

$6(42 \%)$
SÍ BEBIERON

$61(39 \%)$

$94(61 \%)$

\section{Sentimientos de disminución de la capacidad}

de controlar el consumo ( $n=169)$ (n.s.)

NO DISMINUCIÓN

$6(75 \%)$

$63(39 \%)$

SÍ DISMINUCIÓN

$2(25 \%)$

$98(61 \%)$

Aparición de síndrome de abstinencia $(n=166)$ (n.s.)

NO SÍNDROME DE ABSTINENCIA

SÍ SÍNDROME DE ABSTINENCIA

$21(42 \%)$

$34(58 \%)$
$47(42 \%)$

$64(58 \%)$
Criterios evolutivos: consideramos como variables dependientes la efectividad del papel del tratamiento con naltrexona, de las cuáles, la abstinencia es la variable principal y el resto serían variables evolutivas secundarias.

Abstinencia en el consumo a los seis meses valorado por: manifestación del paciente, Manifestación del familiar, control en el aire espirado (siempre que fue posible), control urinario (siempre que sea posible), para considerarse abstinente tendrá que cumplirse, al menos, dos criterios de los anteriores.

Como criterio de recaída se usarán los especificados por Volpicelli et al. (1992) y definidos por nosotros previamente en el apartado anterior: tiempo hasta la primera recaída y hasta primer consumo. Días de consumo durante el tratamiento. Cantidad de alcohol consumida por ocasión. Descenso en el craving. Cambio en las áreas laboral, familiar y psicopatológica en el EuropASI.

El criterio Recaída se ha observado cuando el paciente en alguna de las visitas registramos que ha consumido 5 o más días, o bien que la cantidad de Unidades de Bebida Estándar (UBE) sean iguales o superiores a 5, o la cantidad por ocasión, también sea igual o mayor a 5, o se abandona por causas desconocidas.

Acontecimientos adversos: los efectos adversos se han recogido mediante la valoración de la escala UKU modificada para este trabajo, señalando aquellos que estén posiblemente o probablemente relacionados con la naltrexona. Los acontecimientos adversos graves, se han notificado al Plan Nacional de Farmacovigilancia.

\section{Análisis estadístico:}

Estudio observacional no controlado y multicéntrico descriptivo de las características de la muestra y de los resultados del tratamiento. En este análisis entra- rán todos los sujetos que tomaran al menos una dosis de naltrexona (análisis de intención de tratar). Aquellos sujetos perdidos durante el seguimiento serán considerados recaídas, si no existe constancia de lo contrario.

\section{RESULTADOS}

En el estudio se incluyeron, finalmente 234 pacientes, de los cuales se dispone de datos de evaluación de 173 pacientes. El 47\% procedían de centros de tratamiento del alcoholismo, el resto de centros de salud mental, hospitales y otros (tabla 1).

De 61 de los 234 pacientes no se dispuso de evaluación final, desconociéndose su estado final. Se consideraron válidos 173 pacientes (73,9\%). De éstos 5 abandonaron por efectos adversos, 2 por ineficacia, 5 por no aceptar el programa por parte del paciente, 15 incumplieron el tratamiento y en 12 casos desconocemos las causas (tabla 2).

Como tratamientos de desintoxicación se empleó clormetiazol en 44 pacientes $(25,4 \%)$, benzodiacepinas en $42(24,35)$, Tiapride en $26815 \%)$ y otros fármacos en $27(15,6 \%)$.

Como tratamiento de deshabituación se empleó, además de naltrexona, apoyo psicológico en 123 pacientes $(71,1 \%)$, grupos de autoayuda en 46 pacientes $(26,6 \%)$, terapias conductistas en $35(20,2 \%)$ y terapia de familia en $41(23,7 \%)$.

Se emplearon hipnóticos en 9 sujetos (5,2\%).

El cumplimiento del tratamiento se consideró completo en 94 pacientes $(63,1 \%)$, casi toda (más del $80 \%$ de tomas) en $40(26,8 \%)$, poco cumplimiento en $9(6 \%)$ y errática (menos del $20 \%$ ) en $6(4 \%)$. 
Un 35\% del total de sujetos del grupo de tratamiento con naltrexona recayeron, al considerar los que completaron el tratamiento esta cifra era del $26 \%$ y en los altos cumplidores se reducía a un $14 \%$

Durante el seguimiento 79 pacientes de los 173 que cumplieron la evaluación final presentaron requisitos de recaída, un 45,7\% ( $p<0,005$ límites entre $38,1 \%-53,4 \%$ ) y 140 de la muestra total tuvieron algún consumo de alcohol durante el tratamiento con naltrexona $(59,8 \%)$. Si consideráramos la muestra total de 234 pacientes, los 140 que tuvieron recaídas representarían un 59,8\% (Tabla 2). Los 173 pacientes son los que han manifestado una auténtica intención de tratamiento y son sobre los que se va a trabajar y dar el máximo de información porque son los que hemos podido evaluar por completo.

Hemos analizado las posibles recaídas o no sin incluir los abandonos y comprobamos que los que no recayeron superaron a los que sí lo hicieron, tanto en la muestra global como en la de los pacientes finalizados $61,5 \%$ y $61,9 \%$, respectivamente (tabla 3 )

La sensación de mejoría final sentida tanto por el paciente como por el clínico, definidas como mejorías marcadas o moderadas superan el $86 \%$ entre lo sentido por los pacientes y el 82,3\% entre los clínicos. Los empeoramientos fueron sentidos por el 1,2\% de los pacientes y el 1,9\% de los pacientes (Tabla 4)

Los cambios en las sensaciones relacionadas con el alcohol, fueron diferentes para los que bebieron o no lo hicieron, observamos un mayor deseo de consumo en los que bebieron (61\%). La sensación de disminución de la capacidad de control de consumo fueron superiores entre los que bebieron en alguna ocasión (61\%). Por lo que respecta a aparición de síndrome de abstinencia, fue similar en porcentaje entre los que bebieron y los que no lo hicieron (58\%) (tabla 5).

Los pacientes que han manifestado algún consumo de alcohol, sin cumplir los requisitos de recaída han sido los siguientes: no bebieron nada 69 (40,6\%), bebieron en alguna ocasión 101 (59,4\%), no encontrándose ninguna significación en dichas diferencias.

La implicación familiar durante el tratamiento se valoró en una escala desde alta hasta baja, siendo la alta, la derivada de acudir con el paciente, la familia o la pareja en el $95 \%$ de las entrevistas al menos en 147 casos que respondieron fue de 84 personas $(57,14 \%)$.; la implicación media se dio en 26 casos $(17,6 \%)$ y la baja en 37 casos $(25,1 \%)$.

La tolerancia al tratamiento se evaluó como buena, moderada o mala y los resultados sobre 155 casos fueron de buena en 118 pacientes (76,1\%); moderada en $34(21,9 \%)$ y mala en $3(1,9 \%)$.

Causas del abandono del tratamiento $(n=161)$, en 114 casos no ha habido abandono (70,8\%); efectos secundarios en 5 casos (3,1\%); por ser ineficaz para el paciente en 2 pacientes (1,2\%); No ser bien aceptado por el paciente $5(3,1 \%)$; incumplimiento por parte del paciente $15(9,3 \%)$ y por causas desconocidas 20 $(12,4 \%)$

\section{DISCUSIÓN}

El estudio se planteó como un estudio para valorar una serie de variables, diferentes a las de estudios controlados, es decir valorar un antes y un después de la administración de naltrexona en pacientes alcohólicos tras seis meses de tratamiento, que según Chick en $1999^{18}$, parecen tener la ventaja de controlar algunas variables no evaluadas que puedan repercutir en los resultados.

Debemos valorar que ha habido una pérdida de evaluación final de 61 pacientes, porque creemos que la situación clínica fue menos controlable porque se trataba de pacientes que acudían a la consulta diaria, y su aceptación de la participación en el estudio es de menor exigencia que en los estudios controlados, como ocurrió en los trabajos de Volpicelli et al. ${ }^{13}$.

También debemos opinar que el seguimiento a seis meses fue superior en el tiempo a trabajos clásicos en naltrexona en alcohólicos como los de VolpiceIli et. Al $1992^{13}$ y los de O'Malley et al. $1992^{14}$, que fueron de 12 semanas, más o menos tres meses.

Por lo que respecta a la eficacia, es decir, mantenimiento de la abstinencia al cabo de seis meses después de interrumpir el tratamiento, O'Malley et al. $1996^{19}$, con terapias comportamentales, unos con naltrexona y otros con placebo, tras un mes de interrumpido el tratamiento. Se vio por parte de los autores que era mejor el mantenimiento de tratamiento con naltrexona y apoyos comportamentales, a partir de las 12 semanas.

\section{Tasas de recaída:}

Berg et al. $1996^{20}$ con 6 meses de tratamiento obtuvieron una reducción del $50 \%$ en las tasas de recaída junto a un tratamiento conductual.

Para O'Malley et al. $1995^{21}$ en estudios controlados y con hospitalización parcial durante un mes y tratamiento psicosocial obtuvieron un aumento de los períodos de abstinencia, disminuyendo muy significativamente el consumo alcohólico en un 36\% y reduce en un $50 \%$ las recaídas, comparadas frente a placebo. En otro trabajo, O'Malley et al. $1996^{20}$, tras abandonar la naltrexona al menos 6 meses, vieron que presentaban menos recaídas entre los tratados con naltrexona Un 63\% frente a un 37\% de los que habían recibido placebo.

O'Malley et al. $1992^{14}$ en su estudio con alcohólicos durante 12 semanas de forma aleatoria con dos psicoterapias, prevención de recaídas y de afrontamiento 
con naltrexona o placebo, dieron como resultado que la naltrexona frente al placebo ya que bebían menos, incrementándose los porcentajes de abstinencia y el número de bebidas era inferior.

Volpicelli et al. $1992^{13}$ observaron que los que estuvieron en tratamiento con placebo el $95 \%$ de ellos recayó tras probar el alcohol, mientras que sólo un $50 \%$ de los tratados con naltrexona mantuvo criterios de recaída.

Entre nuestros pacientes podemos considerar que 79 de 173 presentaron unos criterios de recaída un $(45,7 \%)$, próximas a los resultados de los autores contrastados, siendo un $54,3 \%$ los que no las habían presentado.

La recaída incluyendo abandonos fue de un 38,1\% de los pacientes, mientras que los que no recayeron y no abandonaron fueron un $61,9 \%$ de la muestra. Datos superiores a los manifestados por Volpicelli et al. $1997^{15}$

Pudimos ver que la impresión clínica global de los pacientes es muy aceptable, incluso mejor que la de los clínicos con un $86 \%$ de sensación de mejoría, frente a un $82 \%$ observada por los clínicos.

Disminución del craving y de los efectos placenteros del alcohol

Volpicelli et al $1992^{13}$ estudiando a 70 pacientes tratados durante 12 semanas, determinaron en un tratamiento doble ciego naltrexona con placebo, que los que tomaron naltrexona tuvieron menos deseos de beber (craving), bloqueo de los efectos reforzantes positivos del alcohol, con menor sensación de placer y menos días de consumo de alcohol.

Volpicelli et al. $1995^{12}$ sostienen que es un medicamento que reduce las recaídas y el "craving", el número de días de consumo de alcohol y la tasa de recaídas y con pocos efectos secundarios. Al mismo tiempo que los alcohólicos con bajos niveles de craving, alcanzan buenos resultados recibiendo solamente psicoterapia.

En un trabajo entre 70 veteranos tratados en un doble ciego por Volpicelli et al, 199523, 36 de ellos abandonaron la abstinencia. El 80,5\% de éstos informaron sobre los efectos subjetivos de la bebida durante el ensayo. Entre los tratados con naltrexona 7 de 12 frente a los tratados con placebo, informaron que la "euforia" producida por el alcohol fue significativamente menor de lo habitual.

Landabaso et al. 199717 sostuvieron las mismas conclusiones en un estudio controlado con 30 pacientes, viendo que un $40 \%$ del grupo control recaían, mientras que sólo lo hacía un 7\% de los tratados con naltrexona. También un $40 \%$ de los del grupo control mantenía la abstinencia, frente a un $80 \%$ de los tratados con naltrexona.
O'Malley et al. $1996^{20}$ en un estudio entre 16 pacientes tratados con naltrexona frente a 27 tratados con placebo, vieron que los tratados con naltrexona presentaron menos ansias de beber (craving), además de que habían manifestado menos recompensa por el consumo de alcohol, sosteniendo la teoría de que la naltrexona reduce el ansia de beber (craving).

Ciraulo et al. $1997^{24}$ tras repasar múltiples estudios admitieron que la naltrexona consigue disminuir el ansia por la bebida lo que le lleva a alcanzar altos niveles de abstinencia. La eficacia de naltrexona aumenta considerablemente cuando mejora su cumplimiento terapéutico.

En nuestros pacientes observamos que existió un deseo intenso de consumo mayor entre los que bebieron en alguna ocasión en un $61 \%$ frente a un $42 \%$ de los que no bebieron. Mientras que los que no bebieron, no presentaron craving un 58\% de los que no bebieron frente a un 39\% de los que sí lo hicieron.

Los alcohólicos que no bebieron, manifestaron en un $75 \%$ la sensación de que no disminuía su capacidad de controlar el consumo, mientras que los que sí bebieron en alguna ocasión esa capacidad queda muy reducida y se aumenta el porcentaje de ellos que sí sintieron la disminución de controlar el consumo.

\section{Cumplimiento del tratamiento}

Volpicelli et al. $1997^{15}$ tras comparar sus diversos estudios previos, determinaron que la eficacia de la naltrexona aumenta al mejorar el cumplimiento de su tratamiento, al mismo tiempo que los pacientes demostraron ser buenos cumplidores en la toma del mismo. Lo mismo sostienen Ciraulo et al. ${ }^{25}$

Croop et al. $1997^{25}$ en un estudio con 865 pacientes de 40 centros, se distribuyeron aleatoriamente de la siguiente manera 295 pacientes a un grupo control y 570 al grupo de tratamiento con naltrexona determinaron que el 49,2\% de los pacientes del grupo control y un $38,6 \%$ de los del grupo naltrexona, finalizaron el tratamiento.

El cumplimiento del tratamiento de forma estricta fue valorado en un 63,1\% de los pacientes, casi completo en un $26,8 \%$ y poco cumplimiento o errático en un $10 \%$ de los mismos, lo que indica un criterio aceptable de cumplimiento, aspecto que se corresponde con los criterios de Chick $^{19}$.

La tolerancia al tratamiento fue buena en un $76,1 \%$ de los pacientes, moderada en un 21,9\%, lo que habla a favor de la utilización de dicho tratamiento.

Los abandonos del tratamiento con naltrexona entre los que manifestaron una intención de tratamiento fueron de un 29,2\%, lo que nos parece una cifra relativamente baja, para lo que vemos en la clínica habitualmente y las causas de los mismos fueron por sentir efectos secundarios, por considerarlo ineficaz, por no ser bien aceptado por el paciente, el 
incumplimiento puro y duro y por otras razones no especificadas. Lo siguieron un 70,8\%.

Los alcohólicos españoles suelen, a pesar de sus trastornos conductuales, tener una buena implicación familiar en su tratamiento, acudiendo con sus familias a todas las visitas en un $57,1 \%$, y con una implicación media en un $17,6 \%$ y baja o muy escasa en un $25,1 \%$.

Debemos decir que datos como estos no han sido muy evaluados en todos los tratamientos con naltrexona realizados con anterioridad y que nos parecen de sumo interés para mejorar los resultados de los pacientes alcohólicos tratados con naltrexona siempre combinada con tratamientos psicosociales y apoyos terapéuticos diversos.

AGRADECIMIENTOS: a todos los participantes en la recogida de datos del estudio GEODA en toda España, por orden alfabético: Miguel Abad, César Álvarez, Francisco Arias, $M^{a}$ Mercé Barcells, Miguel Ángel Beltran, Santa Blanco, Javier Blanco, Ricardo Calle, Joan Canellas, Bartolomé Cañuelo, Francisco José Castro, Evangelina Claro, Pedro Cuadrado, Juan diez, Fernando Luis Espí, Elvira Fernández, Román Fernández, Jordi Ferré, José Antonio García, Esther García, Aurelio García, Josep Guardia, Jorge Guerola, Juan Francisco Hernández, Ana Isabel Marcos, Juan Ignacio Markez, Juan José Martínez, Jacinto Mosquera, Enriqueta Ochoa, Imanol Querejeta, José Luis Rabadán, Pilar Rojano, Jorge Sánchez, Juan Sanz, Amelia Silva, Miguel Ángel Torres, Francisco Verjano, Gonzalo Vilches.

FINANCIACIÓN: El estudio Geoda fue oficialmente comunicado al Ministerio de Sanidad. El estudio ha contado con el apoyo financiero de Dupont-Pharma

\section{NOTAS}

(1) Nathan PE Outcomes of treatment for alcoholism: current data. Ann. Behav. Med. 1986 8: 40-46.

(2) Froehlich JC, Li TK Recent developments in alcoholism: opioid peptides. Recent. Dev. Alcohol. 1993 11: 187-205.

(3) Fuller RK, Roth HP. Disulfiram for the treatment of alcoholism: an evaluation in 128 men. Ann. Intern. Med. 1979 90: 901-904.

(4) Fuller RK, Branchey L, Brigthwell DR, Derman RM, Emrick LD, Iber EL, et al. Disulfiram treatment of alcoholism: a veterans administration cooperative study. JAMA 1986 256: 1449-1455.

(5) Altshuler HI, Phillips PE, Feinhandler DA. Alteration of ethanol self-administration by naltrexone. Life Sci. 1980 26: 679-688.

(6) Gianoulakis, C. Endogenous opioids and excessive alcohol consumption. J. Psychiatry Neurosci. 1993 18: 148-156.

(7) Aguirre JC, Del Arbol JL, Raya J, et al. (1990) Plasma betaendorphin levels in chronic alcoholics. Alcohol. 1990 7: 409-412.

(8) Gianoulakis C, Krishnan B, Thavundayil J. Enhanced sensitivity of pituitary beta-endorphin to ethanol in subjects at high risk of alcoholism. Arch. Gen. Psychiatry (1996) 53: 250-257.

(9) Froehlich JC. Genetic factors in alcohol self-administration. J. Clin. Psychiatry 561995 (suppl.7): 15-23.
(10) Swift RM. Effect of naltrexone on human alcohol consumption. J. Clin. Psychiatry 561995 (suppl. 7): 24-29.

(11) O'Mara NB, Wesley LC. Naltrexone in the treatment of alcohol dependence. Ann. Pharmacother. 1994 28: 210-211.

(12) Volpicelli JR, Alterman Al, Hayashida M, O'Brien CP Naltrexone in the treatment of alcohol dependence. Arch. Gen. Psychiatry, 1995 49: 876-880.

(13) Volpicelli JR, Clay KL, Watson NT, O'Brien CP. Naltrexone in the treatment of alcoholism: Predicting response to naltrexone. J. Clin. Psychiatry, 561992 (suppl. 7): 39-44.

(14) O'Malley, SS, Jaffe AJ, Chang G., Schottenfeld RS, Meyer RE, Rounsaville B. Naltrexone and coping skills therapy for alcohol dependence. A controlled study. Arch. Gen. Psychiatry (1992) 49: 881-887.

(15) Volpicelli JR, Rhines KC, Rhines JS, Volpicelli LA, Alterman Al, O'Brien CP. Naltrexone and alcohol dependence. Role of subject compliance. Arch. Gen. Psychiatry, 1997 54: 737-742.

(16) Martínez M, Llobell G, Peralba JL, Toral JR. Evaluación de la eficacia de naltrexona en la dependencia alcohólica mediante determinación sérica de transferrina deficiente en carbohidratos. An. Med. Intern. (Madrid) 1997 12: 589-592.

(17) Landabaso MA, Iraurgi I, Sanz J, Fernández B, Ruiz J, JiménezLerma JM, Calle R, Araluce K, Gutiérrez M. Naltrexona más aversivo en alcohólicos refractarios a tratamiento. Psiquiatría Biológica 1997 4: 5-8.

(18) Chick, J. Estudios naturalísticos versus estudios controlados en las recientes investigaciones sobre el tratamiento de la dependencia del alcohol. Adicciones 1999 11: 257-260.

(19) O'Malley SS, Jaffe AJ, Chang G, Rode, Schottenfeld R, Meyer $R E$, Rounsaville B. Six-month follow-up of naltrexone and psychotherapy for alcohol dependence. Arch. Gen. Psychiatry 1996 53: 217-224.

(20) Berg BJ, Pettinati HM, Volpicelli JR. Valoración riesgo-beneficio de la naltrexona en el tratamiento de la dependencia alcohólica (traducido). Drug Safety. 1996 Oct.; 15 (4): 274-282.

(21) O'Malley SS, Croop RS, Wroblewski JM, Volpicelli J. Naltrexone in the treatment of Alcohol Dependence: A combined analysis of two trials. Psychiatric Annals. 1995 Nov; 25: 681-688.

\section{BIBLIOGRAFÍA}

Nathan PE Outcomes of treatment for alcoholism: current data. Ann. Behav. Med. 1986 8: 40-46.

Froehlich JC, Li TK Recent developments in alcoholism: opioid peptides. Recent. Dev. Alcohol. 1993 11: 187205.

Fuller RK, Roth HP. Disulfiram for the treatment of alcoholism: an evaluation in 128 men. Ann. Intern. Med. 1979 90: 901-904

Fuller RK, Branchey L, Brigthwell DR, Derman RM, Emrick $L D$, lber $E L$, et al. Disulfiram treatment of alcoholism: a veterans administration cooperative study. JAMA 1986 256: 1449-1455.

Altshuler HI, Phillips PE, Feinhandler DA. Alteration of ethanol self-administration by naltrexone. Life Sci. 1980 26: 679-688.

Gianoulakis, C. Endogenous opioids and excessive alcohol consumption. J. Psychiatry Neurosci. 1993 18: 148-156.

Aguirre JC, Del Arbol JL, Raya J, et al. (1990) Plasma betaendorphin levels in chronic alcoholics. Alcohol. 1990 7: 409-412. 
Gianoulakis C, Krishnan B, Thavundayil J. Enhanced sensitivity of pituitary beta-endorphin to ethanol in subjects at high risk of alcoholism. Arch. Gen. Psychiatry (1996) 53: 250-257.

Froehlich JC. Genetic factors in alcohol self-administration. J. Clin. Psychiatry 561995 (suppl. 7): 15-23.

$x$ Swift RM. Effect of naltrexone on human alcohol consumption. J. Clin. Psychiatry 561995 (suppl. 7): 24-29.

O'Mara NB, Wesley LC. Naltrexone in the treatment of alcohol dependence. Ann. Pharmacother. 1994 28: 210-211.

Volpicelli JR, Alterman Al, Hayashida M, O'Brien CP Naltrexone in the treatment of alcohol dependence. Arch. Gen. Psychiatry, 1995 49: 876-880.

Volpicelli JR, Clay KL, Watson NT, O'Brien CP. Naltrexone in the treatment of alcoholism: Predicting response to naltrexone. J. Clin. Psychiatry, 561992 (suppl. 7): 3944.

O'Malley, SS, Jaffe AJ, Chang G., Schottenfeld RS, Meyer RE, Rounsaville B. Naltrexone and coping skills therapy for alcohol dependence. A controlled study. Arch. Gen. Psychiatry (1992) 49: 881-887.

Volpicelli JR, Rhines KC, Rhines JS, Volpicelli LA, Alterman Al, O'Brien CP. Naltrexone and alcohol dependence. Role of subject compliance. Arch. Gen. Psychiatry, 1997 54: 737-742.

Martínez M, Llobell G, Peralba JL, Toral JR. Evaluación de la eficacia de naltrexona en la dependencia alcohólica mediante determinación sérica de transferrina deficiente en carbohidratos. An. Med. Intern. (Madrid) 1997 12: 589-592.

Landabaso MA, Iraurgi I, Sanz J, Fernández B, Ruiz J, Jiménez-Lerma JM, Calle R, Araluce K, Gutiérrez M. Naltre- xona más aversivo en alcohólicos refractarios a tratamiento. Psiquiatría Biológica 1997 4: 5-8.

Chick, J. Estudios naturalísticos versus estudios controlados en las recientes investigaciones sobre el tratamiento de la dependencia del alcohol. Adicciones 1999 11: 257-260.

O'Malley SS, Jaffe AJ, Chang G, Rode, Schottenfeld R, Meyer RE, Rounsaville B. Six-month follow-up of naltrexone and psychotherapy for alcohol dependence. Arch. Gen. Psychiatry 1996 53: 217-224.

Berg BJ, Pettinati HM, Volpicelli JR. Valoración riesgo-beneficio de la naltrexona en el tratamiento de la dependencia alcohólica (traducido). Drug Safety. 1996 Oct.; 15 (4): 274-282.

O'Malley SS, Croop RS, Wroblewski JM, Volpicelli J. Naltrexone in the treatment of Alcohol Dependence: A combined analysis of two trials. Psychiatric Annals. 1995 Nov; 25: 681-688.

Volpicelli JR, Clay KL, Watson NT, O'Brien ChP. Naltrexone in the treatment of alcoholism: predicting response to naltrexone. J clin Psychiatry. 1995; 56 (7): 39-44.

Volpicelli JR, Watson NT, King AC, sherman CE, O'Brien C. Effect of naltrexone on alcohol "high" in alcoholics. Am. J. Psychiatry (1995) 152; 613-615.

Ciraulo AM, Alpert N, Franko KJ. Naltrexone for the treatment of alcoholism. American Family Physician 1997; 56 (3): 803-806.

Croop RS, Fralkner EB, Labriola DF. The safety profile of naltrexone in the treatment of alcoholism. Results from a multicenter usage study. Arch. Gen. Psychiatry 1997; 54: 1130-1135. 
